\title{
Where to Next? The Impact of COVID-19 on Human-Robot Interaction Research
}

\author{
DAVID FEIL-SEIFER, University of Nevada, Reno \\ KERSTIN S. HARING, University of Denver \\ SILVIA ROSSI, University of Naples Federico II \\ ALAN R. WAGNER, Pennsylvania State University \\ TOM WILLIAMS, Colorado School of Mines
}

The COVID-19 pandemic will have a profound and long-lasting impact on the entire scientific endeavor. Scientists already are adapting research programs to adapt to changes in what is prioritized-and what is possible; educators are changing the way that the next generation of researchers are trained, and flagship conferences in many fields are being cancelled, postponed, and fundamentally transformed.

These broad-reaching changes are particularly impactful to human-oriented domains such as human-robot interaction (HRI). Because in-person human-subject experiments can take a year or more to conduct, the research we will see published in the field in the immediate future may appear to be "business as usual," with accounts of laboratory studies with large numbers of in-person participants. The research currently being performed, however, is of course a different story entirely. Studies that were under way when the current crisis began will be truncated, resulting either in work that cannot be published or in work whose true impact is difficult to accurately assess. Yet HRI research performed in the coming years will be changed in fundamentally different ways; the inability to perform-or expect future performance of-in-person human subjects research, especially research involving tactile or multiparty interaction, will change both the dominant methodological techniques employed by HRI researchers and the very research questions that the field chooses to-and is able to-address.

These challenges demand that HRI researchers identify precisely how the field can maintain research quality and impact while the ability to conduct human-subject studies is severely impaired for an undetermined amount of time. A natural inclination may be simply to wait the crisis out in the hope of a speedy return to normalcy; however, in this article, we argue that the community can also take this opportunity to reevaluate and refocus how research in this field is conducted and how students are mentored in ways that will yield benefits for years to come after the current crisis has ended.

CCS Concepts: • Computer systems organization $\rightarrow$ Robotics; Social and professional topics $\rightarrow$ Computing education; Computational science and engineering education; Assistive technologies; Remote medicine;

Additional Key Words and Phrases: Human-robot interaction, research, COVID-19 impact

\footnotetext{
Authors' addresses: D. Feil-Seifer, University of Nevada, Reno, 1664 North Virginia Street MS-171, Reno, NV 895570171; email: dave@cse.unr.edu; K. S. Haring, University of Denver, 2155 East Wesley Avenue, Denver, CO; email: kerstin. haring@du.edu; S. Rossi, University of Naples Federico II, DIETI, via Claudio, 80125 Napoli, Italy; email: silvia.rossi@ unina.it; A. R. Wagner, Pennsylvania State University, Hammond Building, University Park, PA; email: alan.r.wagner@ psu.edu; T. Williams, Colorado School of Mines, 1600 Illinois Street, Golden, CO; email: twilliams@mines.edu.

Permission to make digital or hard copies of part or all of this work for personal or classroom use is granted without fee provided that copies are not made or distributed for profit or commercial advantage and that copies bear this notice and the full citation on the first page. Copyrights for third-party components of this work must be honored. For all other uses, contact the owner/author(s).

(c) 2020 Copyright held by the owner/author(s).

2573-9522/2020/06-ART1 \$15.00

https://doi.org/10.1145/3405450
} 


\section{ACM Reference format:}

David Feil-Seifer, Kerstin S. Haring, Silvia Rossi, Alan R. Wagner, and Tom Williams. 2020. Where to Next? The Impact of COVID-19 on Human-Robot Interaction Research. ACM Trans. Hum.-Robot Interact. 10, 1, Article 1 (June 2020), 7 pages.

https://doi.org/10.1145/3405450

\section{IMPACTS OF COVID-19 ON THE HUMAN-ROBOT INTERACTION RESEARCH PRAXIS}

Human-robot interaction (HRI) is a growing research area, with an increasing number of research methodologies being employed in published work. Within the past 5 years of the International Conference on Social Robotics, ${ }^{1}$ for example, around $40 \%$ of papers presented the results of inlaboratory human-subject experiments, $20 \%$ of papers presented the results of online (crowdsourced) human-subject experiments, $20 \%$ of papers evaluated robot behaviors without humansubject evaluations (e.g., using simulations or evaluation on previously collected datasets), and the remaining $20 \%$ of papers presented contributions that did not require traditional evaluations, such as position papers, innovative robot capabilities demonstrated as proofs of concept, or new techniques for robot design. These proportions are expected to dramatically change over the next 2 years as in-person laboratory experimentation becomes untenable.

In light of the rapid spread of COVID-19, universities have hastily moved much of their operations, including classes, research, and conferences, online. As the dust on this effort slowly settles, it becomes increasingly apparent that the suspension of travel and closure of in-person contact at universities worldwide will have a lasting impact on academic research in HRI. Studies that were in the middle of data collection had to be stopped, and others that were about to begin could not take place. To date, it is unclear when academic in-person studies will be safe again.

For researchers who evaluate the effects of physically present social robots, this presents several challenges. For example, these researchers must now choose between either publishing much smaller datasets than anticipated and potentially not reaching statistically meaningful results due to small sample sizes, or delaying the data evaluation and publication of their research until further data can be collected. For students and researchers who are facing deadlines, this is a difficult choice to make, as the traditional publication model highly values research validated with in-person studies with large sample sizes.

These concerns will manifest differently for different types of research questions. Studies that explore proximal human group reactions to robot behavior [9], ecologically valid reactions to robots by groups of humans [16], group reactions to aberrant human behavior toward robots [2], tactile interactions of humans and robot [7], or robot facilitation of proximal human-human interactions [13] may not be feasible for the immediate future. Not only have many universities forbidden such work for the time being, but it is also unclear if human subjects would be willing to participate. Research on these types of questions may have to simply wait until this situation subsides.

The impacts of COVID-19 on the HRI research praxis present an interesting challenge for the field. They also pose an interesting mentorship opportunity for advisors to young researchers and students. We have known as a field that there are many ways to experimentally approach a single research question. We have typically chosen the easiest experiment to implement to examine that research question. However, we now need to study these questions in the safest possible

\footnotetext{
${ }^{1}$ We choose to focus on ICSR specifically, as the authors of this article represent the lead organizing committee for ICSR 2020 and have ready access to data for past iterations of that conference.
} 
manner. That may require us to examine the same research question in a different fashion, both experimentally and from a planing perspective. Researchers, faculty, and students need to consider contingency plans for HRI studies that minimize in-person contact. Research grant proposals will need to present contingencies for experimental studies that require in-person contact. However, the potential opportunity to mentors is that by implementing and maximizing research flexibility and contingencies, students may, in fact, receive better HRI training. They will be exposed, at least in theory, to a variety of experimental approaches to answer the same research question, as well as learning and experiencing the planning, executing, and adjusting often necessary in experimental HRI research.

Universities and laboratories will gradually reopen, presenting researchers and laboratory directors with the challenge of safely resuming such in-person studies. Determination of safety precautions for participants, researchers, and research assistants must be taken to conduct in-person studies. Some studies may be amended to current social distancing requirements. Replication of Solomon Asch's famous conformity study with robots by Vollmer et al. [18] could be conducted in a manner that maintains social distance [18]. Similarly, the study by Tan et al. [17] of the bystander interventions of robot abuse could also, perhaps, have been performed at a distance [17]. Because these studies only require the participant to observe a robot or the behavior of a confederate toward a robot, it is likely that they can be successfully performed at a social distance. However, requirements that people wear masks in public may complicate the ability to read facial expressions [11].

Many of these concerns could of course be addressed by moving studies online, although the validity of online user studies can wildly vary depending on the nature of the research question being addressed $[1,20]$. Online experiments are already used extensively in the field, such as in work by Jackson et al. [8], who use online experimentation to study morally sensitive topics and employ experimental designs in ways that are fundamentally infeasible in the laboratory, or in work by Kwon et al. [10], who use online studies to examine trust between a human and a robot. However, it is critical to note that these concerns may well apply to online studies as well, if they require coordination between multiple research personnel to film videos and/or operate the robots used in those videos. Accordingly, HRI researchers conducting any sort of empirical research (including filming videos to move experiments online) may well need to follow the same safety guidelines as those seeking to conduct in-person research. Moreover, online responses by human subjects may be different from how these subjects respond in ecologically valid environments [14].

Currently, an institutional review board, as an administrative body established to protect the rights and welfare of human research subjects recruited to participate in research activities, ensures that the methods used to conduct such research are ethical. HRI studies are often classified as minimal risk, indicating that subjects are exposed to no risk beyond everyday life. However, current everyday life risk might include being exposed to a highly contagious virus. To ensure the safety of human subjects and researchers, research guidelines should contain measures to minimize the risk or exposure to pathogens. These guidelines might also be subject to change as more is known about how long the virus remains active on certain surfaces. Some basic guidelines would include minimizing direct contact of researchers and subjects (ensuring social distancing policies of a minimum 6-foot (2-m) distance); a protocol of personal hygiene for the subject and researcher (e.g., washing hands thoroughly before and after the study and each interaction, face masks for research personnel); and a cleaning protocol for all surfaces that are touched, not just the robots and equipment (e.g., including door handles, keyboards, mice, table surfaces, pens).

Although the preceding guidelines may help ensure that empirical HRI research is conducted safely, the concerns delineated previously suggest that other types of research may be more 
productive and feasible during a pandemic that still follow rigorous scientific methods, advance the field of HRI, and contribute to the body of knowledge in HRI. Submissions investigating and developing the theoretical underpinnings necessary to create broadly interactive and social robots will be particularly valuable to the field. Most domains of scientific enquiry advance through continual crafting, expansion, refinement, and critique of empirically grounded theories. And although there have indeed been several efforts toward crafting theories of HRI, especially in areas such as human-robot trust $[4,19]$ and communication [5], one critique that has been leveled at the field is that it suffers from a preponderance of one-off studies that are neither grounded in nor contribute to overarching theories of interaction. This moment in history may well be a fruitful time for HRI researchers to produce surveys of the past few decades of work, meta-analyses of those collected works, and papers developing fundamentally new theories of HRI brought to light through those meta-analyses.

Based on these expectations, we suggest that HRI researchers focus their research efforts toward (1) online, observational, and bystander studies; (2) in-person experiments with low sample sizes that suggest new avenues for research exploration and clearly indicate the need for follow-up with a larger sample size; (3) surveys and meta-analyses; (4) proposal of fundamental new theories of interaction without novel data collection; and (5) technical contributions to artificial intelligence and robotics that will benefit interactive robots, even if they cannot yet be evaluated in interactive contexts.

\section{IMPACTS OF COVID-19 ON HRI RESEARCH TOPICS}

Although COVID-19 is negatively impacting HRI researchers' ability to conduct research using traditional methodologies, it is also revealing promising new areas of inquiry. Researchers in the broader robotics community have hypothesized that one consequence of the pandemic will be to alter the conversation around robots by highlighting that while robotics and automation may be eliminating jobs and exacerbating income inequality, they are also critical for enabling a global economy that is robust to pandemics, by enabling work to continue while limiting human-tohuman contact and potential spread of the disease [21].

Social robots specifically have a clear role to play in a society that is trying to fulfill critical services while decreasing human-to-human contact, and that is seeking to provide social support to isolated and vulnerable populations. Although social robots cannot completely replace humans and the feeling of being close to another human being, they can support people in their efforts of increasing physical distance from others, reducing direct interactions when they are unnecessary (e.g., by supporting distant communication and telemedicine, providing telepresence facilities [3]), support efforts in distance learning [6] (e.g., especially for children through age-appropriate robot interactions), and provide social and emotional support and companionship to vulnerable people who would otherwise be alone [12]. Research that makes these social robots feasible is therefore crucial at this time.

Moreover, service robotics will be critical for helping workers with applications that are not intended to require interaction (e.g., by taking on delivery or waste removal tasks). However, few considerations have been made concerning workers who need to share space with robots, even if not directly interacting with them, and how robots' actions in space-sharing contexts will impact human perceptions, actions, and task performance, as well as novel privacy concerns that may arise [15].

It is thus anticipated that in the aftermath of COVID-19, HRI will experience increased attention, focused on developing robots that can be deployed in future pandemic events while mitigating the possible negative impact of those robots during periods of normal operation. Based on these 
expectations, we suggest that the current crisis raises a host of exciting and important new research questions, oriented around topics such as the following:

- Social robots for testing, treatment, and decontamination in medical settings

- Social robots for at-home companionship, tutoring, and education

- Socially aware behaviors for facilitating contact-free delivery

- Robot teleoperation interfaces and interactions with teleoperated robots

- Robots for facilitating long-distance social interaction and telemedicine

- Socially aware navigation assistance for telepresence

- Robot proxemics and interaction fostering social distancing guidelines

- Methodologies for performing and evaluating HRI research, both practically and ethically, during periods of enforced isolation

- Pedagogical techniques for online HRI education.

\section{IMPACTS OF COVID-19 ON STUDENT DEVELOPMENT}

The HRI community must also identify how best to operate as a community to ensure that the benefits traditionally sustained by in-person experiences are not lost. This is especially important as conferences are moved online, postponed, or cancelled, or when in-person laboratory research is not possible. Those who are likely to be hit hardest are our students, who are among the most socially and economically vulnerable members of our communities.

Students benefit from in-laboratory experience in ways that are difficult to measure. From actually working with robots in a hands-on fashion, or near-peer mentoring, to incidental contact with faculty who are not the students' advisors, there is definite value to being present in a laboratory. Similarly, student development is a critical yet often overlooked aspect of in-person conference attendance. Students contribute and benefit from conference participation by interacting and presenting in front of an audience of seasoned researchers, attending conference presentations from their peers, and developing cohorts with other students. Attending and presenting at a conference can lead to valuable learning experiences, professional opportunities, and collaborations. With conference interactions minimized as well, faculty and mentors should work toward making sure that students are able to set up a professional network with peers and faculty who share their research interests within and outside their own organizations.

In addition to research flexibility, teaching flexibility must also be considered. Student training and learning opportunities often are centered on being in a laboratory and working directly with robots, and students benefit from the near-peer interactions and mentorship available. Online studies will likely abstract away much of the important experiences of working with robots in person. It might be beneficial for students to get additional experience with in-person robots once it is safe to do so. For laboratory leaders, this could mean giving students access to facilities and robots one student at a time, such as with a staggered or distributed laboratory access. Tied to faculty enabling individual student experiences, the challenge arises of not overextending a single mentor's time commitment yet giving each student the in-person learning experience with robots. This also challenges organizations and institutions to support their faculty and student mentors appropriately and enable them to adjust their teaching.

It is therefore imperative that while this crisis persists, our field seeks effective methods to continue to foster student development. For example, events like virtual community meetups and happy-hours can allow students to interact with senior researchers informally. The community may also hold mentoring sessions in which senior researchers meet with students from outside their universities to offer comments about students' public papers and presentations, and virtual laboratory visits in which students present to and brainstorm with fellow HRI graduate students 
from other universities. It may also be necessary for the HRI community to put greater effort toward community-level publicity, marketing, blogging, and social media presence to ensure that the broader community learns about new research from students in the field, and to promote virtual job fairs with hiring departments and companies, to maximize the hire rates for students in our field. Even though these changes will not replace the missed opportunity of attending conferences in person, it may soften the impact of the crisis on our students and provide new and unique opportunities for development.

\section{CALL FOR ACTION}

Given the new restrictions put on daily life by social distancing requirements, it would be easy to become dismayed by assuming that the study of HRI is in a state of paralysis. This crisis, however, presents an opportunity for the members of this community to reflect on their research practices, both to conform to the new guidelines and to improve the field overall.

With in-person studies currently presenting a challenge, more research energy and investment can be directed to the development of better survey instruments, more comprehensive and overarching theories, much-needed meta-analyses, and comprehensive surveys of the field. In addition, some attention must be redirected to developing social robots to support clinicians and essential workers, as well as how HRI can enhance online education, and provide companionship for isolated people. For any in-person research study, it is crucial to identify a contingency plan if social distancing has to resume where the study is being conducted. This certainly affects research practices, but it should also impact how we request research funding and how those funding requests are reviewed. Finally, given that it is not currently safe to congregate people for an in-person study, perhaps a reexamination of individual user studies is warranted.

The field of HRI can also change its mentorship practices to better fit the current times. With in-laboratory activities reduced or suspended all together, students should be mentored in the multiple ways that a research question can be examined by experimental practices, both in person and using alternative means. With the cancellation of in-person conferences and travel in general, we must take care to find ways for students to expand their professional networks beyond their own laboratories and universities. In some ways, when in-laboratory activities are suspended, it might become as natural to collaborate with someone from another institution as with one at your own. This could greatly expand the professional network for students in the HRI field.

This crisis will likely fundamentally disrupt the field of HRI for some time. This community should take the time afforded by this crisis to reinvent and reorganize the experimental methodologies and mentoring practices that have traditionally been prized to continue to advance this vital field.

\section{REFERENCES}

[1] Frederik Aust, Birk Diedenhofen, Sebastian Ullrich, and Jochen Musch. 2013. Seriousness checks are useful to improve data validity in online research. Behavior Research Methods 45, 2 (2013), 527-535.

[2] Zachary Carlson, Louise Lemmon, MacCallister Higgins, David Frank, Roya Salek Shahrezaie, and David Feil-Seifer. 2019. Perceived mistreatment and emotional capability following aggressive treatment of robots and computers. International fournal of Social Robotics 11, 5 (Oct. 2019), 727-739. DOI : https://doi.org/10.1007/s12369-019-00599-8

[3] Amedeo Cesta, Gabriella Cortellessa, Andrea Orlandini, and Lorenza Tiberio. 2016. Long-term evaluation of a telepresence robot for the elderly: Methodology and ecological case study. International fournal of Social Robotics 8 (2016), 421-441. DOI : https://doi.org/10.1007/s12369-016-0337-z

[4] Ewart J. de Visser, Marieke M. M. Peeters, Malte F. Jung, Spencer Kohn, Tyler H. Shaw, Richard Pak, and Mark A. Neerincx. 2020. Towards a theory of longitudinal trust calibration in human-robot teams. International fournal of Social Robotics 12 (2020), 459-478.

[5] Chad Edwards, Autumn Edwards, Patric R. Spence, and David Westerman. 2016. Initial interaction expectations with robots: Testing the human-to-human interaction script. Communication Studies 67, 2 (2016), 227-238. 
[6] N. T. Fitter, N. Raghunath, E. Cha, C. A. Sanchez, L. Takayama, and M. J. Mataric. 2020. Are we there yet? Comparing remote learning technologies in the university classroom. IEEE Robotics and Automation Letters 5, 2 (2020), 2706-2713.

[7] Kerstin Sophie Haring, Katsumi Watanabe, David Silvera-Tawil, Mari Velonaki, and Yoshio Matsumoto. 2015. Touching an Android robot: Would you do it and how? In Proceedings of the 2015 International Conference on Control, Automation, and Robotics. IEEE, Los Alamitos, CA, 8-13.

[8] Ryan Blake Jackson, Tom Williams, and Nicole Smith. 2020. Exploring the role of gender in perceptions of robotic noncompliance. In Proceedings of the 2020 ACM/IEEE International Conference on Human-Robot Interaction. 559-567.

[9] Takayuki Kanda, Masahiro Shiomi, Zenta Miyashita, Hiroshi Ishiguro, and Norihiro Hagita. 2009. An affective guide robot in a shopping mall. In Proceedings of the 4th ACM/IEEE International Conference on Human Robot Interaction. $173-180$.

[10] Minae Kwon, Erdem Biyik, Aditi Talati, Karan Bhasin, Dylan P. Losey, and Dorsa Sadigh. 2020. When humans aren't optimal: Robots that collaborate with risk-aware humans. In Proceedings of the 2020 ACM/IEEE International Conference on Human-Robot Interaction (HRI'20). ACM, New York, NY, 43-52. DOI : https://doi.org/10.1145/3319502.3374832

[11] Rosalind W. Picard. 2000. Affective Computing. MIT Press, Cambridge, MA.

[12] Lihui Pu, Wendy Moyle, Cindy Jones, and Michael Todorovic. 2018. The effectiveness of social robots for older adults: A systematic review and meta-analysis of randomized controlled studies. Gerontologist 59, 1 (2018), e37-e51. DOI : https://doi.org/10.1093/geront/gny046

[13] Nina Riether, Frank Hegel, Britta Wrede, and Gernot Horstmann. 2012. Social facilitation with social robots? In Proceedings of the 2012 7th ACM/IEEE International Conference on Human-Robot Interaction (HRI'12). IEEE, Los Alamitos, CA, 41-47.

[14] Paul Robinette, Ayanna M. Howard, and Alan R. Wagner. 2017. Effect of robot performance on human-robot trust in time-critical situations. IEEE Transactions on Human-Machine Systems 47, 4 (2017), 425-436.

[15] Silvia Rossi, Alessandra Rossi, and Kerstin Dautenhahn. 2020. The secret life of robots: Perspectives and challenges for robot's behaviours during non-interactive tasks. International fournal of Social Robotics. Published online. April 23, 2020. DOI : https://doi.org/10.1007/s12369-020-00650-z

[16] Selma Sabanovic, Marek P. Michalowski, and Reid Simmons. 2006. Robots in the wild: Observing human-robot social interaction outside the lab. In Proceedings of the 9th IEEE International Workshop on Advanced Motion Control. IEEE, Los Alamitos, CA, 596-601.

[17] Xiang Zhi Tan, Marynel Vázquez, Elizabeth J. Carter, Cecilia G. Morales, and Aaron Steinfeld. 2018. Inducing bystander interventions during robot abuse with social mechanisms. In Proceedings of the 2018 ACM/IEEE International Conference on Human-Robot Interaction. 169-177.

[18] Anna-Lisa Vollmer, Robin Read, Dries Trippas, and Tony Belpaeme. 2018. Children conform, adults resist: A robot group induced peer pressure on normative social conformity. Science Robotics 3, 21 (2018), eaat7111.

[19] Alan R. Wagner, Paul Robinette, and Ayanna Howard. 2018. Modeling the human-robot trust phenomenon: A conceptual framework based on risk. ACM Transactions on Interactive Intelligent Systems 8, 4 (2018), 1-24.

[20] Claire Wladis and Jason Samuels. 2016. Do online readiness surveys do what they claim? Validity, reliability, and subsequent student enrollment decisions. Computers \& Education 98 (2016), 39-56.

[21] Guang-Zhong Yang, Bradley J. Nelson, Robin R. Murphy, Howie Choset, Henrik Christensen, Steven H. Collins, Paolo Dario, et al. 2020. Combating COVID-19-The role of robotics in managing public health and infectious diseases. Science Robotics 5, 40 (2020), eabb5589. DOI : https://robotics.sciencemag.org/content/5/40/eabb5589.full.pdf

Received May 2020; accepted June 2020 